\title{
HIV-1 Nef targets restriction factors
}

\section{ce}

\section{Nef targets}

... SERINC3

and SERINC5, redirecting them from the plasma membrane to endosomes.

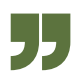

The HIV-1 accessory protein Nef has several roles during infection, including enhancing virion infectivity. This process involves the endocytic machinery of the host cell, but the exact molecular mechanism is unknown. Now, two new studies reveal that Nef targets two new restriction factors, serine incorporator 3 (SERINC3) and SERINC5, redirecting them from the plasma membrane to endosomes.

Restriction factors are host proteins that counteract viral replication and are usually inhibited by viral proteins. Usami et al. examined whether Nef targets an unidentified restriction factor by conducting a proteomic analysis of virions produced by cells infected with wild-type versus Nef-defective HIV-1. They identified SERINC3, a member of a family of transmembrane proteins, as being consistently present in Nef-defective virus but absent from wild-type HIV-1. In

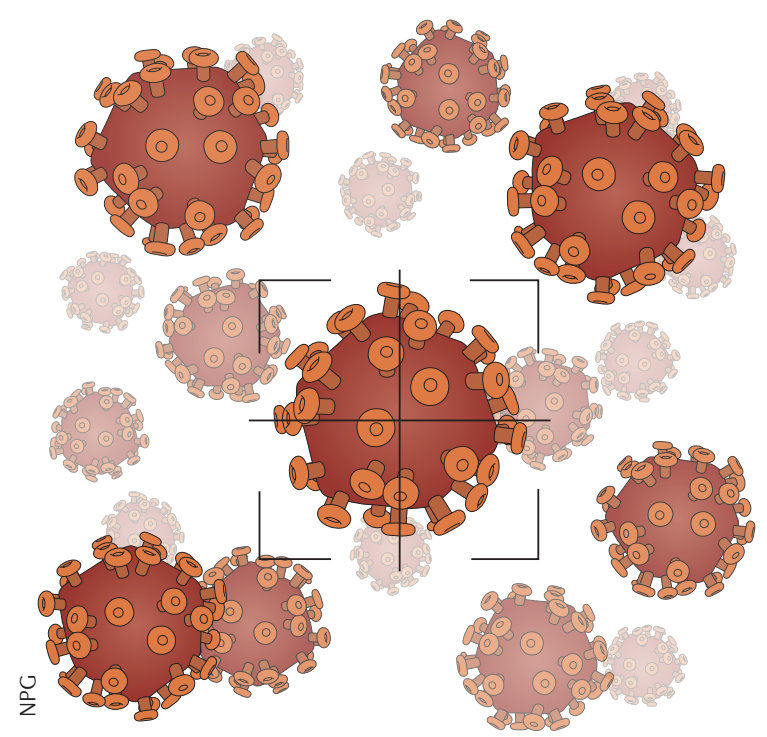

another study, Rosa et al. infected 31 human cell lines with wild-type versus Nef-defective HIV-1 and, using a global transcriptome analysis, correlated SERINC5 expression with Nef-dependent changes in viral infectivity. Notably, both groups then investigated the role of other SERINC proteins, with Usami et al. identifying SERINC5 and Rosa et al. identifying SERINC3 as being involved in the decrease in HIV-1 infectivity observed for Nef-defective HIV-1.

To validate their findings, Rosa et al. used CRISPR-Cas9 to disrupt SERINC5 in human cell lines and peripheral blood mononuclear cells, which resulted in a notable increase in infectivity of Nef-defective HIV-1. This effect was abolished following SERINC5 complementation, and SERINC5 overexpression inhibited HIV-1 infection even when viruses were capable of expressing Nef.

Usami et al. used short interfering RNAs (siRNAs) and CRISPR-Cas9 to respectively downregulate or disrupt SERINC3, SERINC5 or both, which resulted in increased infectivity of Nef-defective HIV-1. Interestingly, simultaneous targeting of SERINC3 and SERINC5 enhanced the individual effects, suggesting that both proteins mediate this process. Furthermore, overexpression of SERINC5 reduced the infectivity of Nef-defective HIV-1, and this effect was counteracted by Nef overexpression.

Both studies then tagged SERINC3 or SERINC5 to visualize their distribution following infection. When Nef is absent, both proteins localize in the plasma membrane and are incorporated into budding virions; however, in the presence of Nef, the proteins are no longer present in the plasma membrane and their incorporation into virions is disrupted. Notably, when cells were infected with wild-type virus, Rosa et al. visualized SERINC5 and Nef in late endosomes, which was dependent on the expression of the host protein AP2 - an adaptor protein involved in clathrin-dependent endocytosis - providing a link between the activity of Nef and the endocytic machinery.

Finally, both studies examined how SERINC3 and SERINC5 incorporation into virions reduces HIV-1 infectivity and found that the proteins do not inhibit viral fusion with the target cell, but rather seem to prevent the enlargement of the fusion pore. Although this mechanism requires confirmation, it could impair the passage of the viral capsid core into the cytoplasm of the infected cell.

Collectively, these studies establish SERINC3 and SERINC5 as novel HIV-1 restriction factors and demonstrate that Nef targets these proteins by redirecting them to endosomes, where they can no longer be incorporated into budding virions. Cláudio Nunes-Alves

ORIGINAL RESEARCH PAPERS Rosa, A. et al. HIV-1 Nef promotes infection by excluding SERINC5 from virion incorporation. Nature http:// dx.doi.org/doi:10.1038/nature15399 (2015) | Usami, Y. et al. SERINC3 and SERINC5 restrict HIV-1 infectivity and are counteracted by Nef. Nature http://dx.doi.org/10.1038/nature15400 (2015)

FURTHER READING Campbell, E. M. \& Hope, T. J. HIV-1 capsid: the multifaceted key player in HIV-1 infection. Nat. Rev. Microbiol. 13, 471-483 (2015) | Freed, E. O. HIV-1 assembly, release and maturation Nat. Rev. Microbiol. 13, 484-496 (2015) 